\title{
A Level Set Approach to Segmenting A Deforming Myocardium from Dynamically Acquired SPECT Projection Data
}

\author{
Florin Neacsu, Rostyslav Boutchko, Member IEEE, Archontis Giannakidis, and Grant T. Gullberg, Fellow, IEEE
}

\begin{abstract}
Dynamic cardiac single photon emission computed tomography (SPECT) offers an effective way for observing fundamental physiological functions of organs and could aid in the early diagnosis of cardiovascular disease, in particular, for those patients with minimal disease. This would improve the chances of recovery by initiating appropriate therapy and an altered life style. To make dynamic cardiac SPECT viable with present clinical scanners methods need to be developed that reconstruct time activity curves from dynamically moving organs representing the change of tracer concentration as a function of time from projection data acquired from slowly rotating gamma cameras. This type of data analysis faces the challenge of modeling both rigid and non-rigid body deformation as well as modeling of a time varying tracer concentration. In the work presented here, we develop methods for segmenting the beating heart using an approach based upon level sets, which can deal naturally with topological changes. A variational formulation of the level set method was implemented. This allowed the inclusion of a priori information and was computationally efficient. The algorithm was first evaluated with simulated dynamic cardiac image data. The MCAT phantom was used to generate data containing 32 time frames over one cardiac cycle. Each frame had a matrix size of $64 \times 64 \times 32$ voxels with a resolution of 6.25 $\mathrm{mm}$. Starting with an initial estimate of the boundary, the algorithm then converged to an accurate segmentation of the deforming heart. The initial estimate was not important and we could segment simultaneously both interior and exterior boundaries. This algorithm forms the foundation for the segmentation of the boundary of the deforming myocardium directly from projection data.
\end{abstract}

\section{INTRODUCTION}

An estimated 80,000,000 American adults (one out of three) suffer from one or more types of cardiovascular disease (CVD), with half of them estimated to be age 60 or older. Nearly 2,400 Americans die of CVD each day, which gives an average of one death every 37 seconds. CVD overall death toll accounted for about $56 \%$ of all deaths in 2005 [1].

Manuscript received November 21, 2010. This work was supported by NIH under Grant No. R01 HL50663 and R01EB00121 and by the Director, Office of Science, Office of Biological and Environmental Research of the US Department of Energy under contract DE-AC02-05CH11231.

F. Neacsu was with the Lawrence Berkeley National Laboratory, Berkeley CA, USA and is now a student at Ecole superieure de Chimie Physique Electronique, Lyon, France (e-mail: fneacsu@ gmail.com).

R. Boutchko, A. Giannakidis, and G. T. Gullberg are with the Lawrence Berkeley National Laboratory, Berkeley CA, USA.
Dynamic single photon emission computed tomography is a nuclear imaging modality [2] that offers an effective way for observing fundamental physiological functions of organs. For example, it offers the ability to quantify myocardial perfusion of a tracer injected into the blood [4]. The observations involve the quantification of temporal changes of the concentration of the radionuclide tracer in the targeted organs. Data acquisition is usually performed with slow rotating gamma cameras. Model-based data analysis is used to estimate physiological compartment parameters representing the wash-in and wash-out of the radiopharmaceutical from the organ of interest. It is anticipated that dynamic cardiac SPECT could aid in the early diagnosis of cardiovascular disease and in particular for those patients with minimal disease. This could improve the chances of recovery by initiating therapy and an altered life style.

The overall objective of this research is to implement algorithms that are able to reconstruct three dimensional (3D) dynamically moving organs and time activity curves (TAC) representing the change in concentration of a radiopharmaceutical in the organ. This estimation of dynamic parameters that model the deformation of the organ, as a function of time, and the change of the tracer concentration, as a function of time, from projections, is referred to as 5D tomography [3].

To segment and define boundaries of the beating heart, we use level set methods. Level set methods have been used with success in a variety of domains, image processing and medical imaging, in particular, being among them [7]. Promising results obtained in positron emission tomography (PET) [10] encouraged us to utilize this framework.

This study is an extension of our work in four dimensional (4D) tomography [4] reconstructing dynamically deforming organs. It faces the challenge of modeling both rigid and non-rigid body deformation, together with the difficult task of modeling of a time varying tracer concentration from tomographic projections. This paper is organized as follows. In Section 2 we give a short introduction to level set methods. In Section 3 we describe our methods for segmenting cardiac boundaries from images of a 
deforming myocardium and segmenting them from the projections of a deforming object. In Section 4 we present simulation results. Finally, Section 5 presents conclusions and future work.

\section{LEVEL SETS}

The segmentation of the heart is accomplished by determining boundaries of deforming organs. Suppose one has to divide a domain of $\Re^{n}$ into multiple subdomains. In order to do that, an interface must be defined to bind the subdomains. Note that the subdomains are subsets of $\Re^{n}$ while the interface is a subset of $\Re^{n-1}$. In an explicit interface representation, one needs to define explicitly each point belonging to the interface, whereas an implicit interface representation defines the interface as the isocontour of a function. An interface $C$ can be represented by the zero level set of a function $\phi: C(t)=\{(x, y) \mid \phi(t, x, y)=0\}$. To add dynamics to the evolution of the interface, an evolution equation was introduced by Osher and Sethian [6], the Eulerian formulation of which is given by

$$
\frac{\partial \phi}{\partial t}=|\nabla \phi| F
$$

where $F$ is called the speed function, which can depend on a number of factors: with one of them being the curvature [7]. This formulation has the important advantage of handling topological changes naturally. However, it can develop 'shocks' and 'rarefaction fans' [5] when using simple difference schemes because it is a hyperbolic equation. In order to deal with this issue, a common usage is to initialize (and re-initialize, after a number of steps) the function $\phi$ as a signed distance function; hence rendering the equation parabolic.

The re-initialization step can be computationally costly. Our algorithm is similar to the variational level set method presented in [8]. This formulation has the advantage of incorporating shape-prior information. It also does not require a re-initialization of $\phi$.

\section{METHODS}

\section{A. Segmenting Cardiac Boundaries from Images of a Deforming Myocardium}

In this section we present a curve evolution method for segmenting cardiac boundaries from images of a deforming myocardium.

Let us define an energy function unifying external energy, internal energy, and energy terms incorporating prior information as follows
$E(\phi) \equiv \mu P(\phi)+\lambda L_{g}(\phi)+v A_{g}(\phi)+\xi E_{s}(\phi)+\alpha E_{t}(\phi)$

where $\mu, \lambda, v, \xi$, and $\alpha$ are parameters controlling the relative weights of the energy terms, $g$ is the edge indicator function, $L_{g}(\phi)$ expresses the length of the zero level curve of $\phi, A_{g}(\phi)$ is the weighted area of

$\Omega_{\phi}^{-}=\{(x, y) \mid \phi(x, y)<0\}, \quad E_{s}(\phi) \quad$ imposes spatial smoothness, and $E_{t}(\phi)$ assures temporal smoothness [9]. The gradient flow, which minimizes $E$, is given by $\partial \phi / \partial t=-\partial E / \partial \phi$. The right hand side of this equation can be expressed by calculus of variation in terms of $\phi$ and its derivatives [11]. This yields a partial differential equation (PDE) in the form $\partial \phi / \partial t=F(\phi)$. This PDE gives the evolution of the level set function $\phi$ whose zero level will evolve towards the boundary of the heart. Temporal smoothness issues were not treated in this study.

Next we explain the terms mentioned above. A signed distance function $\phi$ satisfies $|\nabla \phi|=1$ and any function

$f$ satisfying $|\nabla f|=1$ is a signed distance function. The metric

$$
P(\phi)=\int_{\Omega} \frac{1}{2}(|\nabla \phi|-1)^{2} d x d y
$$

was introduced to measure the deviation of $\phi$ from a signed distance function [8]. The edge detector function is defined by

$$
g(|\nabla I(x, y)|) \equiv \frac{1}{1+\left|\nabla G_{\sigma}(x, y) * I(x, y)\right|^{2}},
$$

where $I$ is an image and $G_{\sigma}$ is the Gaussian kernel with standard deviation $\sigma$ :

$$
G_{\sigma}(x, y)=\sigma^{-1 / 2} e^{-\left|x^{2}+y^{2}\right| / 4 \sigma} .
$$

The function $g(|\nabla I(x, y)|)$ is positive in homogeneous regions and zero at the edges. The surface integral of a function $f$ over the boundary $\partial \Omega$ is given by

$$
\int_{\Omega} f\left(\vec{x}^{\prime}\right) \delta\left(\phi\left(\vec{x}^{\prime}\right)\right)\left|\nabla \phi\left(\vec{x}^{\prime}\right)\right| d \vec{x},
$$


where $\delta$ is the Dirac function. Hence, in $\Re^{2}$ we define $L_{g}(\phi)$ as

$$
L_{g}(\phi) \equiv \int_{\Omega} g \delta(\phi)|\nabla \phi| d x d y
$$

The volume integral of a function $f$ over the interior region $\Omega^{-}$is

$$
\int_{\Omega} f\left(\vec{x}^{\prime}\right)\left(1-H\left(\phi\left(\vec{x}^{\prime}\right)\right) d \vec{x}^{\prime},\right.
$$

where $H$ is the Heaviside function. Hence, in $\Re^{2}$, we define

$$
A_{g}(\phi) \equiv \int_{\Omega} g H(-\phi) d x d y
$$

These two terms, $L_{g}(\phi)$ and $A_{g}(\phi)$, drive the zero level set towards the object boundaries. $E_{s}(\phi)$ is defined as the sum of length of all curves

$$
E_{s}(\phi) \equiv \sum_{k=1}^{K} \int_{C_{k}} d s
$$

where $k \in\{1,2, \ldots, K\}$ represents the time step of a dynamic tomographic reconstruction and $C_{k}$ is the zero level set of $\phi$ at time $k$.

\section{B. Segmenting Boundaries from Projections}

In this section we present a curve evolution method for segmenting boundaries of a deforming object from projections of the deforming object.

Let $C$ denote the object boundaries and $\beta$ the different intensities. With these notations we can express the projection at a certain angle and at a certain time as follows

$$
y_{i}^{k}(C, \beta)=\int h_{i}(x) f(x, C, \beta) d x+e_{i},
$$

with $i=1, \ldots, M$ the angles of the acquisition, $h_{i}$ the projection kernel, and $e_{i}$ the error assumed to be Poisson noise. In order to reconstruct $C$ and $\beta$ we use the expression for the energy

$$
E=-\log (p(y \mid Y(C, \beta)))+\int_{C} d C .
$$

By minimizing the first term, we maximize the likelihood of the observed data, and by minimizing the second term, which represents the length of $C$, we obtain smooth curves. Taking into account temporal boundary smoothness and temporal intensity smoothness the previous energy function becomes

$$
E=E_{d}+\lambda_{s} E_{s}+\lambda_{t} E_{t}+\lambda_{i} E_{i},
$$

where $E_{d}$ is the data fidelity term; $E_{s}$ is the spatial boundary smoothness term; $E_{t}$ is the temporal boundary smoothness term; $E_{i}$ is the temporal intensity smoothness term; and $\lambda_{s}, \lambda_{t}$ and $\lambda_{i}$ are constant weight parameters. For an imaging problem the number of photons emitted from each image pixel is a Poisson random variable and each measurement can be treated as the summation of these Poisson variables. We can set up the likelihood function as the joint probably mass function of all Poisson distributed random variables and by taking the logarithm of this likelihood function we can express $E_{d}$ as

$$
E_{d}=\sum_{k=1}^{K} \sum_{i=1}^{M}\left(Y_{i}^{k}-y_{i}^{k} \ln Y_{i}^{k}\right)+\text { cons } \tan t
$$

with $i=1, \ldots, M$ the acquisition angles and $k=1, \ldots, K$ the time frame. The constant part of the logarithm of the likelihood function is ignored as it does not depend on the variables we are looking for. The spatial boundary smoothness term is expressed as previously,

$$
E_{s}(\phi) \equiv \sum_{k=1}^{K} \int_{C_{k}} d s
$$

A two step coordinate descent algorithm was used. First we assumed $C$ fixed and minimize $E$ with respect to $\beta$ and secondly we assumed $\beta$ fixed and minimized $E$ with respect to $C$.

\section{RESUlTS}

\section{A. Segmenting Cardiac Boundaries from Images of a Deforming Myocardium}

Using the MCAT phantom [12] we generated a full cardiac cycle, representing the blood pools of beating left and right ventricles with lung motion. This resulted in 32 time frames of $64 \times 64 \times 32$. Figure 1 shows the interior (blue) and exterior (red) segmentation of slice 11 from the time step 5 at 4 different iterations steps. 

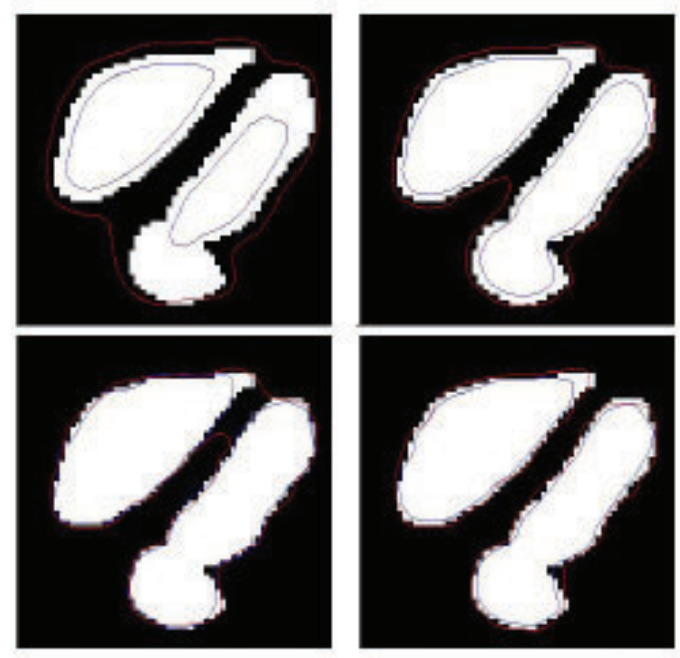

Figure 1. Segmentation results form images of a deforming myocardial blood pool. From left to right, top to bottom, results are shown for iteration 50, 200, 800, and 1500 .

\section{B. Segmenting Boundaries from Projections}

Taking into account only the first two terms of the energy term in Eq. (13), the two step gradient descent algorithm was able to reconstruct both $C$ and $\beta$ for a simple numerical phantom. We simulated projections of a square of a given uniform intensity which was used to test the algorithm. Figure 2 shows on the left side our phantom, a square of intensity 10 and our initial guess, a circle of intensity -2 . The initial guess does not influence the outcome of the algorithm: it could be of any shape and size. On the right side of Figure 2, we see the result of the algorithm. Due to the superposition of the two figures, we had to lower the alpha component, which alters the figures intensity scale. The intensity was accurately reconstructed, obtaining in the end a uniform intensity of 9.8 .


Figure 2: Left: initial guess; Right: reconstructed shape and intensity

\section{CONCLUSION AND FUTURE WORK}

In this work, we presented a level set method to segment the beating heart. The proposed algorithm is computationally efficient and has been tested on both simulated images and real data. In the near future our efforts will be oriented towards incorporating temporal smoothness and intensity dynamics constraints into the algorithm.

This algorithm forms the foundation for our future work involving the segmentation of the boundary of the deforming myocardium directly from projection data and allows the incorporation of mechanical models of the myocardium that can be used as a prior in the estimation of the time evolution of tracer time activity curves.

\section{REFERENCES}

[1] Heart Disease and Stroke Statistics 2009 Update, American Heart Association. Available at http://www.americanheart.org.

[2] G. T. Gullberg, B. W. Reutter, A. Sitek, J. S. Maltz, T. F. Budinger, "Dynamic single photon emission computed tomography - Basic principles and cardiac applications," Phys. Med. Biol., vol. 55, pp. R111-R191, 2010.

[3] B. Feng, P. H. Pretorius, T. H. Farncombe, S. T. Dahlberg, M. V. Narayanan, M. N. Wernick, A. M. Celler, J. A. Leppo and M. A. King, "Simultaneous assessment of cardiac perfusion and function using 5dimensional imaging with Tc-99m teboroxime," J. Nucl. Cardiol., vol. 13, pp. 354-61, 2006.

[4] B. W. Reutter, G. T. Gullberg, R. Butchko, K. Balakrishnan, E. H. Botvinick and R. H. Huesman, "Fully 4-D dynamic cardiac SPECT image reconstruction using spatiotemporal B-spline voxelization," in 2007 IEEE Nucl Sci Symp Med Imag Conf Record, pp. 4217-4221, 2007.

[5] S. Osher and R. Fedkiw, "Level set methods and dynamic implicit surfaces," Applied Mathematical Sciences, Springer, New-York, USA, vol. 153, pp. 3-4,23, 2003.

[6] S. Osher, J. A. Sethian, "Fronts propagating with curvature dependent speed - algorithms based on Hamilton-Jacobi formulations," J. Comp. Phys., vol. 79, pp. 12-49, 1988.

[7] J. A. Sethian, Level Set Methods and Fast Marching Methods Evolving Interfaces in Computational Geometry, Fluid Mechanics, Computer Vision, and Material Science, Cambridge University Press 1999.

[8] C. Li, C. Xu, C. Gui, M. D. Fox, "Level set evolution without re-initialization - A new variational formulation, Proceedings of the 2005 IEEE CVPR, 2005.

[9] Y. Shi and W. C. Karl, "Tomographic reconstruction of dynamic objects," Proceedings of SPIE-IS\&T Electronic Imaging, SPIE, vol.5016, 2003.

[10]T. F. Chan, H. Li, X-C Tai, "Level set method for positron emission tomography," Int. Journal of Biomedical Imaging, vol. 2007, Article ID 26950, doi:10.1155/2007/26950. 
[11]L. Evans, Partial Differential Equations, Providence: American Mathematical Society, 1998.

[12] P. H. Pretorius, M. A. King, B. M. W. Tsui, K. J. LaCroix and W. Xia, "A mathematical model of motion of the heart for use in generating source and attenuation maps for simulating emission imaging," Med. Phys., vol. 26, pp. 2323-32, 1999 